\title{
Non-Schedulability Conditions for Off-line Scheduling of Real-Time Systems Subject to Precedence and Strict Periodicity Constraints
}

\author{
Patrick MEUMEU YOMSI \\ INRIA Rocquencourt \\ Domaine de Voluceau BP 105 \\ 78153 Le Chesnay Cedex - France \\ Email: patrick.meumeu@inria.fr
}

\author{
Yves SOREL \\ INRIA Rocquencourt \\ Domaine de Voluceau BP 105 \\ 78153 Le Chesnay Cedex - France \\ Email: yves.sorel@inria.fr
}

\begin{abstract}
Classical off-line approaches based on preemption such as RM (Rate Monotonic), DM (Deadline Monotonic), EDF (Earliest Deadline First), LLF (Least Laxity First), etc, give schedulability conditions but most of the time assuming on the one hand that all the tasks are independent, and on the other hand, that the first instances of all tasks are released at the same time. We are interested in hard real-time systems subject to precedence and strict periodicity constraints, i.e. such that for all instances of each task, the release time and start time are equal. For such systems, it is mandatory to satisfy these constraints. In this paper we give non-schedulability conditions in order to restrict the study field of all systems of tasks to only potentially schedulable systems.
\end{abstract}

\section{Introduction}

We address here hard real-time applications found in the domain of automobile, avionic, mobile robotic, telecommunication, etc, where it is mandatory to satisfy the real-time constraints otherwise dramatic consequences occur [1]. Such applications, based on automatic control and/or signal processing algorithms, are usually specified with block-diagrams. They are composed of functions producing and consuming data, and each function has a strict period. Consequently, in this paper we study the problem of scheduling tasks, onto a single computing resource, i.e. a monoprocessor, each of them corresponding to a function, while satisfying a successor/predecessor relation in addition to their strict periods. Furthermore, since we aim hard real-time, off-line scheduling approaches are better suited because they guarantee a deterministic behavior for the applications.

Classical off-line approaches based on preemption, such as RM (Rate Monotonic) [2], DM (Deadline Monotonic) [3], EDF (Earliest Deadline First) [4], LLF (Least Laxity First) [5], etc, use the Liu \& Layland model [6]. This model allows to give schedulability conditions but assuming on the one hand that all the tasks are independent in that the requests for a certain task do not depend on the initiation or the completion of requests of other tasks, and on the other hand, that the first instances of all tasks are released at the same time. With this model, the release time and the start time of any instance may not be equal for each task during its execution. Actually, because we are in the monoprocessor case, and because we are interested in systems subject to precedence and strict periodicity constraints, two tasks cannot use the processor at the same time. Indeed, strict periodicity constraint imposes for all instances of each task the release time and the start time to be equal. Clearly, this is not the case for the classical model. Furthermore, the start time of any instance of any task should not occurs while the processor is occupied by an already scheduled task.

In this paper, we give non-schedulability conditions in order to restrict the study field of all systems of tasks to only potentially schedulable systems. To achieve this goal we use the extention, with preemption, of our previous model [7] that is well suited to the applications we are interested in.

Afterwards, to clearly distinguish the specification level and its associated model we are interested in, we will use the term operation instead of the commonly used "task" [8] which is too closely related to the implementation level.

The paper is structured as follows: Section 2 describes the model and gives notations used throughout this paper. Section 3 gives non-schedulability conditions to restrict the study field of all systems of tasks to only potentially schedulable systems when seeking a valid schedule thanks on the one hand to properties on the strict periods, and on the other hand to properties on the worst case execution time (WCET) of the tasks. We conclude and propose future works in section 4.

\section{Model}

The model depicted in figure 1 is an extension, with preemption, of our previous model [7] for systems with 
precedence and strict periodicity constraints executed on a single processor.

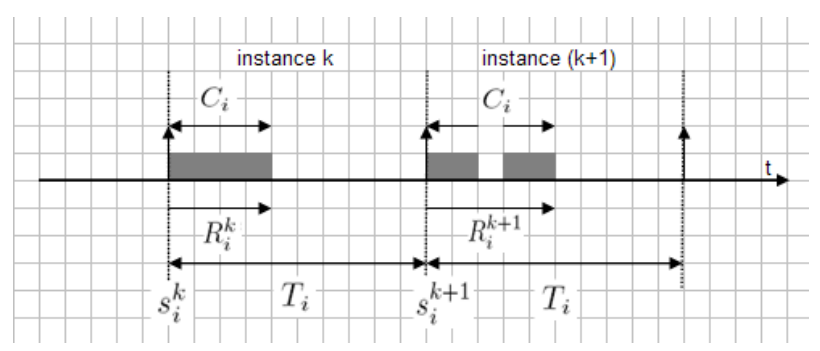

Figure 1. Model

Here are the notations used in this model assuming all temporal values are multiple of the period of the processor clock:

$\tau_{i}$ : An operation

$C_{i}$ : WCET of operation $\tau_{i}, C_{i} \leq T_{i}$

$T_{i}$ : Period of operation $\tau_{i}$

$s_{i}^{0}:$ Start time of the first instance of operation $\tau_{i}$

$s_{i}^{k}=s_{i}^{0}+(k-1) T_{i}$ : Start time of the $k^{t h}$ instance of operation $\tau_{i}$

$R_{i}^{k}$ : Response time of the $k^{\text {th }}$ instance of operation $\tau_{i}$

$R_{i}$ : Worst-case response time of operation $\tau_{i}$

$T_{i} \wedge T_{j}$ : The greatest common divisor of $T_{i}$ and $T_{j}$, when $T_{i} \wedge T_{j}=1, T_{i}$ and $T_{j}$ are co-prime

$\tau_{i} \prec \tau_{j}: \tau_{i} \longrightarrow \tau_{j}, \tau_{i}$ is a predecessor of $\tau_{j}$

We assume a single processor, and denote $V$ the set of systems of operations. Each system consists in a given number of operations, subject to precedence and strict periodicity constraints. Each operation $\tau_{i}$ of a system in $V$ consists in a pair $\left(C_{i}, T_{i}\right): C_{i}$ its WCET and $T_{i}$ its period. The precedence constraints are given by a partial order on the execution of the operations: $\tau_{i} \prec \tau_{j}$ means $\tau_{j}$ cannot start before $\tau_{i}$ is completed. The strict periodicity constraint means that two successive instances of an operation are exactly separated by its period: $s_{i}^{k+1}-s_{i}^{k}=$ $T_{i} \quad \forall k \in \mathbb{N} \quad \forall i \in\{1, \cdots, n\}$. In this model the start time is always equal to the release time, contrary to the Liu \& Layland's classical model, and for each operation, each instance should be completed before the next one starts.

It is fundamental to note that, because of the strict periodicity constraint and the fact that we are in the monoprocessor case, any two instances of two operations of the system cannot start their executions at the same time.

\section{Study field restriction}

Firstly, we eliminate all the systems such that start times of any two instances of any two operations are identical. This will be achieved thanks only to properties on the strict periods of the operations, using the Bezout theorem. This is formally expressed through both theorems given in section 3.1. Secondly, we eliminate all the systems such that the start time of any instance of an operation occurs while the processor is occupied by an already scheduled operation thanks to properties on WCET of the operations. This is formally expressed through the theorem given in section 3.2. These theorems give nonschedulability conditions in order to restrict the study field of all systems of tasks to only potentially schedulable systems.

\subsection{Strict periodicity restriction Theorem 1}

Given a system of $n$ operations in $V$, if there are two operations $\tau_{i}=\left(C_{i}, T_{i}\right)$ and $\tau_{j}=\left(C_{j}, T_{j}\right)$ with $\left(\tau_{i} \prec \tau_{j}\right)$ starting their executions respectively at the dates $s_{i}^{0}$ and $s_{j}^{0}$ such that

$$
T_{i} \wedge T_{j}=1
$$

then the system is not schedulable. Moreover, any additional assumption (for example preemption and idle times) on the system intending to satisfy all the constraints does not have any interest in this case.

\section{Proof}

Let $\tau_{i}=\left(C_{i}, T_{i}\right)$ and $\tau_{j}=\left(C_{j}, T_{j}\right)$ be two operations of a system in $\mathrm{V}$ starting their executions respectively at the dates $s_{i}^{0}$ and $s_{j}^{0}$ such that: $T_{i} \wedge T_{j}=1$.

Notice that $s_{j}^{0} \geq s_{i}^{0}$ because $\tau_{i} \prec \tau_{j}$.

Let:

$$
\gamma=s_{j}^{0}-s_{i}^{0}
$$

If $\gamma=0$, the result is immediate because the first start times of the two operations are then identical. This is not possible because several operations cannot use the processor at the same time.

Let us suppose from now that $\gamma \neq 0$, then:

$$
\exists(\alpha, \beta) \in \mathbb{Z}^{2} \quad \text { such that } \alpha T_{i}=\beta T_{j}+\gamma
$$

Indeed, $T_{i} \wedge T_{j}=1$ implies:

$$
\exists(m, n) \in \mathbb{Z}^{2} \text { such that } m T_{i}-n T_{j}=1(C f . \text { Bezout })
$$
i.e.,

$$
\exists(m, n) \in \mathbb{Z}^{2} \text { such that } \quad(m \gamma) \cdot T_{i}-(n \gamma) \cdot T_{j}=\gamma
$$

Let:

$$
\left\{\begin{array}{l}
\alpha_{0}=(m \gamma) \\
\beta_{0}=(n \gamma)
\end{array}\right.
$$

It is clear that $\left(\alpha_{0}, \beta_{0}\right)$ is a particular solution of (3). The expressions (3) and (4) give:

$$
\left\{\begin{array}{rlll}
\left(\alpha-\alpha_{0}\right) \cdot T_{i}-\left(\beta-\beta_{0}\right) \cdot T_{j} & = & 0 & (*) \\
\alpha_{0} T_{i}-\beta_{0} T_{j} & = & \gamma & (* *)
\end{array}\right.
$$

(*) can be rewritten as : $\left(\alpha-\alpha_{0}\right) \cdot T_{i}=\left(\beta-\beta_{0}\right) \cdot T_{j}$ Thus we have:

$$
\left\{\begin{array}{l}
T_{i} \mid\left(\beta-\beta_{0}\right) \cdot T_{j} \\
T_{i} \wedge T_{j}=1
\end{array}\right.
$$

Hence $T_{i} \mid\left(\beta-\beta_{0}\right)$ i.e.,

$$
\exists k \in \mathbb{N} \text { such that } \beta-\beta_{0}=k T_{i}
$$

Now, replacing $\beta-\beta_{0}$ by its value in $(*)$, we obtain: 


$$
\alpha-\alpha_{0}=k T_{j}
$$

Then:

$$
(\alpha, \beta)=\left(\alpha_{0}+k T_{j}, \beta_{0}+k T_{i}\right)
$$

is the general form of all the solutions of (3).

Given any $k \in \mathbb{N}$, at all the following dates $t_{i, j}^{k}$ the start times of instances of operations $\tau_{i}$ and $\tau_{j}$ are identical:

$$
\begin{aligned}
t_{i, j}^{k} & =s_{i}^{0}+\left(\alpha_{0}+k T_{j}\right) \cdot T_{i} \\
& =s_{i}^{0}+\left(m\left(s_{j}^{0}-s_{i}^{0}\right)+k T_{j}\right) \cdot T_{i} \\
& =s_{j}^{0}+\left(\beta_{0}+k T_{i}\right) \cdot T_{j} \\
& =s_{j}^{0}+\left(n\left(s_{j}^{0}-s_{i}^{0}\right)+k T_{i}\right) \cdot T_{j}
\end{aligned}
$$

and, thus the strict periodicity constraint is violated. Consequently the system is not schedulable.

Since it is not possible to prevent this situation, any other additional assumption on the system intending to satisfy all the constraints is useless.

\section{N.B.}

In the expression (3), the choice of the integers $\alpha$ and $\beta$ can be made in $\mathbb{N}$ to meet the fact that time is positive.

In fact, if (3) is satisfied and $\alpha$ or $\beta$ is negative, we just have to consider $h_{0} \in \mathbb{N}$ large enough such that:

$$
\left\{\begin{array}{l}
\alpha_{1}=\alpha+h_{0} T_{j} \in \mathbb{N} \\
\beta_{1}=\beta+h_{0} T_{i} \in \mathbb{N}
\end{array}\right.
$$

and thus:

$$
(3) \Longleftrightarrow \alpha_{1} T_{i}-\beta_{1} T_{j}=\gamma
$$

In the remainder of this paper, we will no longer care about this detail because it is easy to be solved.

\section{Example 1}

Let $A$ and $B$ be two operations of a system in $\mathrm{V}$ with the characteristics defined in table 1 below:

\section{Table 1. Characteristics of example 1}

\begin{tabular}{c|c|c|c|} 
& $C_{i}$ & $T_{i}$ & $s_{i}^{0}$ \\
\hline$A$ & 1 & 4 & 0 \\
\hline$B$ & 1 & 7 & 2 \\
\hline
\end{tabular}

We notice that $4 \wedge 7=1$ and conclude thanks to theorem 1 that this system is not schedulable. Indeed, at the date $t=16$ in figure 2 the two operations $A$ and $B$ start their executions simultaneously. Consequently we cannot schedule them without violating the strict periodicity constraint of one of the two operations.

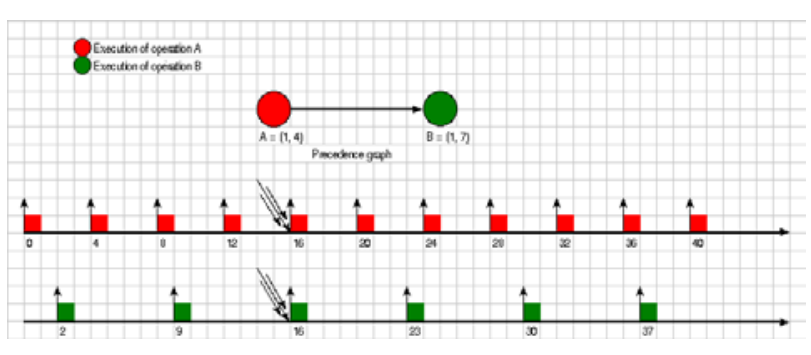

\section{Figure 2. System with 2 operations such} that: $T_{i} \wedge T_{j}=1$

\section{Theorem 2}

Given a system of $n$ operations in $V$, if there are two operations $\tau_{i}=\left(C_{i}, T_{i}\right)$ and $\tau_{j}=\left(C_{j}, T_{j}\right)$ with $\left(\tau_{i} \prec \tau_{j}\right)$ starting their executions respectively at the dates $s_{i}^{0}$ and $s_{j}^{0}$ such that

$$
T_{i} \wedge T_{j} \mid\left(s_{j}^{0}-s_{i}^{0}\right)
$$

then the system is not schedulable. Moreover any additional assumption on the system intending to satisfy all the constraints does not have any interest in this case.

\section{Proof}

Let $\tau_{i}=\left(C_{i}, T_{i}\right)$ and $\tau_{j}=\left(C_{j}, T_{j}\right)$ be two operations of a system in $V$ starting their executions respectively at the dates $s_{i}^{0}$ and $s_{j}^{0}$ such that: $T_{i} \wedge T_{j} \mid\left(s_{j}^{0}-s_{i}^{0}\right)$.

Notice once more that $s_{j}^{0} \geq s_{i}^{0}$ because $\tau_{i} \prec \tau_{j}$. Let:

$$
\gamma=s_{j}^{0}-s_{i}^{0}
$$

We assume $\gamma \neq 0: C f$. proof of Theo.1, then:

$$
\exists(\alpha, \beta) \in \mathbb{Z}^{2} \text { such that } \alpha T_{i}=\beta T_{j}+\gamma
$$

Indeed,

let us assume $T_{i} \wedge T_{j}=\lambda \quad\left(\lambda \in \mathbb{N}^{*}\right)$, then it exists $T_{i}^{1}, T_{j}^{2} \in$ $\mathbb{N}^{*}$ such that:

$$
\left\{\begin{array}{l}
T_{i}=\lambda T_{i}^{1} \\
T_{j}=\lambda T_{j}^{2} \\
T_{i}^{1} \wedge T_{j}^{2}=1
\end{array}\right.
$$

$T_{i}^{1} \wedge T_{j}^{2}=1$ implies:

$$
\exists(m, n) \in \mathbb{Z}^{2} \text { such that } m T_{i}^{1}-n T_{j}^{2}=1(\text { Cf. Bezout }) .
$$

By multiplying each side of (9) by $\lambda$, we obtain:

$$
\begin{array}{cc}
m \cdot\left(\lambda T_{i}^{1}\right)-n \cdot\left(\lambda T_{j}^{1}\right)=\lambda \\
\text { i.e., } \quad m T_{i}-n T_{j}=\lambda
\end{array}
$$

Thus, (8) and (10) give:

$$
(\alpha-m) T_{i}-(\beta-n) T_{j}=\gamma-\lambda
$$

However, we assumed $\lambda \mid \gamma$, i.e.,

$$
\exists p \in \mathbb{N}^{*} \quad \text { such that } \quad \gamma=p \lambda
$$


We then have:

$$
\begin{array}{rc} 
& (\alpha-m) T_{i}-(\beta-n) T_{j}=(p-1) \lambda \\
\text { i.e., } \quad(\alpha-m) T_{i}^{1}-(\beta-n) T_{j}^{2}=p-1
\end{array}
$$

By multiplying each side of (9) by $(p-1)$, we obtain:

$\exists(m, n) \in \mathbb{Z}^{2}$ such that $m(p-1) T_{i}^{1}-n(p-1) T_{j}^{2}=p-1$

The expressions (12) and (13) give:

$$
\exists(m, n) \in \mathbb{Z}^{2} \quad \text { such that } \quad(\alpha-m p) T_{i}^{1}=(\beta-n p) T_{j}^{2}
$$

In this last relation, we have:

$$
\left\{\begin{array}{l}
T_{i}^{1} \mid(\beta-n p) \cdot T_{j}^{2} \\
T_{i}^{1} \wedge T_{j}^{2}=1
\end{array}\right.
$$

Thus $T_{i}^{1} \mid(\beta-n p)$ i.e.,

$$
\exists k \in \mathbb{N}^{*} \quad \text { such that } \quad \beta-n p=k T_{i}^{1}
$$

By replacing this expression in the relation (13), we obtain:

$$
\alpha-m p=k T_{j}^{2}
$$

Consequently, the general form of the solutions of (8) is given by:

$$
(\alpha, \beta)=\left(m \frac{s_{j}^{0}-s_{i}^{0}}{T_{i} \wedge T_{j}}+k \frac{T_{j}}{T_{i} \wedge T_{j}}, n \frac{s_{j}^{0}-s_{i}^{0}}{T_{i} \wedge T_{j}}+k \frac{T_{i}}{T_{i} \wedge T_{j}}\right)
$$

Thus, given any $k \in \mathbb{N}$, at all the following dates $t_{i, j}^{k}$ the start times of instances of operations $\tau_{i}$ and $\tau_{j}$ are identical:

$$
\begin{aligned}
t_{i, j}^{k} & =s_{0}^{i}+\left[m\left(s_{j}^{0}-s_{i}^{0}\right)+k T_{j}\right] \frac{T_{i}}{T_{i} \wedge T_{j}} \\
& =s_{0}^{j}+\left[n\left(s_{j}^{0}-s_{i}^{0}\right)+k T_{i}\right] \frac{T_{j}}{T_{i} \wedge T_{j}}
\end{aligned}
$$

and, thus the strict periodicity constraint is violated. Consequently the system is not schedulable.

Since it is not possible to prevent this situation, any other additional assumption on the system intending to satisfy all the constraints is useless.

\section{Example 2}

Let $A$ and $B$ be two operations of a system in $V$ with the characteristics defined in table 2 :

Table 2. Characteristics of example 2

\begin{tabular}{c|c|c|c|} 
& $C_{i}$ & $T_{i}$ & $s_{i}^{0}$ \\
\hline$A$ & 1 & 4 & 0 \\
\hline$B$ & 1 & 6 & 6 \\
\hline
\end{tabular}

We notice that $4 \wedge 6=2$ and $2 \mid 6$. We can conclude thanks to theorem 2 that this system is not schedulable.

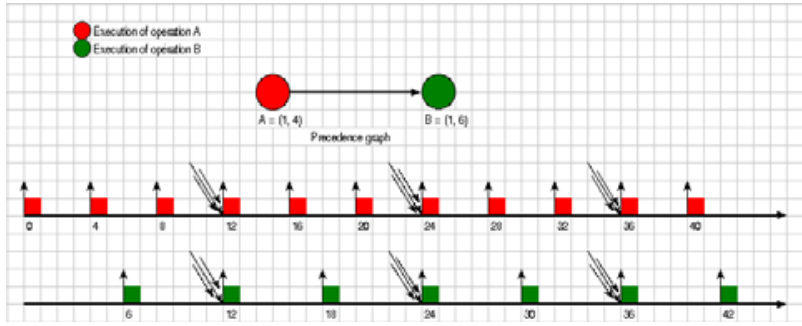

Figure 3. System with 2 operations such that: $T_{i} \wedge T_{j} \mid\left(s_{j}^{0}-s_{i}^{0}\right)$

Indeed, at the dates $t=12, t=24, \cdots$ in figure 3 the two operations $A$ and $B$ start their executions simultaneously. Consequently we cannot schedule them without violating the strict periodicity constraint of one of the two operations.

Theorems 1 and 2 give conditions of non schedulability for systems subject to strict periodicity constraints when both previous relations on the strict periods hold. Moreover, any additional assumption on the system would be useless because of the identical start times of two instances of at least two operations.

We denote by $\Omega_{\lambda}$ the sub-set of $V$ excluding the cases where the strict periods of the operations verify both previous relations, see figure 4 .

$$
\begin{array}{r}
\Omega_{\lambda}=\left\{\left\{\left(C_{i}, T_{i}\right)\right\}_{1 \leq i \leq n} \in V / \forall i, j \in\{1, \cdots, n\}\right. \\
\left.\exists \lambda>1, T_{i} \wedge T_{j}=\lambda \text { and } \lambda \nmid\left(s_{j}^{0}-s_{i}^{0}\right)\right\}
\end{array}
$$

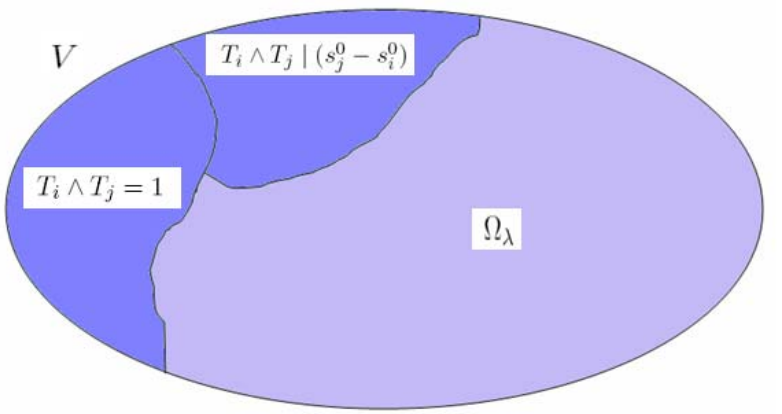

Figure 4. $\Omega_{\lambda}$ : Restriction of the study field

\subsection{WCET restriction}

Because of the precedence constraints, it has been shown in [9] that given two operations $\tau_{i}=\left(C_{i}, T_{i}\right)$ and $\tau_{j}=\left(C_{j}, T_{j}\right)$ :

$$
\tau_{i} \prec \tau_{j} \Longrightarrow T_{i} \leq T_{j}
$$

Thus, the operations must be scheduled in an increasing order of their periods.

Now, we assume any operation of the system may only be preempted by those already scheduled [1], [2] relatively to $\prec$. 
We call $U_{p}$ the $p^{\text {th }}$ temporary load factor of the processor $(1 \leq p \leq n)$ for a system of $n$ operations $\left\{\tau_{i}=\right.$ $\left.\left(C_{i}, T_{i}\right)\right\}_{1 \leq i \leq n}$ in $\Omega_{\lambda}$.

$$
U_{p}=\sum_{i=1}^{p} \frac{C_{i}}{T_{i}}
$$

That system will be said to be potentially schedulable if and only if:

$$
U_{n} \leq 1
$$

From now we assume (20) is always satisfied. A potential schedule $\mathcal{S}$ of a system is given by a list of the start times of the first instance of all the operations:

$$
\mathcal{S}=\left\{\left(s_{1}^{0}, s_{2}^{0}, \cdots, s_{n}^{0}\right)\right\}
$$

The start times $s_{i}^{k}(k \geq 1,1 \leq i \leq n)$ of the other instances of operation $\tau_{i}$ are directly deduced from the first one. The response time $R_{i}^{k}$ of the $k^{t h}$ instance of operation $\tau_{i}=\left(C_{i}, T_{i}\right)$ is the time elapsed between its start time $s_{i}^{k-1}$ and its end time. This latter takes into account the preemption thus,

$$
R_{i}^{k} \geq C_{i} \quad \forall k
$$

We call $R_{i}$ the worst response time of operation $\tau_{i}$, defined as the maximum of the response times of all its instances.

These definitions enable us to say that, to satisfy the strict periodicity, any operation $\tau_{i}=\left(C_{i}, T_{i}\right)$ of a potentially schedulable system in $\Omega_{\lambda}$ must satisfy:

$$
R_{i} \leq T_{i} \quad \forall i \in\{1, \cdots, n\}
$$

We say that a system in $\Omega_{\lambda}$ has one overlapping when the start time of any instance of a given operation occurs while the processor is occupied by an already scheduled operation. Such systems are not schedulable, and we express that through the following theorem.

\section{Theorem 3}

Given a system of $n$ operations in $\Omega_{\lambda}$, if there are two operations $\tau_{i}=\left(C_{i}, T_{i}\right)$ and $\tau_{j}=\left(C_{j}, T_{j}\right)$ with $\left(\tau_{i} \prec \tau_{j}\right)$ starting their executions respectively at the dates $s_{i}^{0}$ and $s_{j}^{0}$ such that

$$
\exists \alpha, \beta \in \mathbb{N} \text { and } 0 \leq\left(s_{j}^{0}+\beta T_{j}\right)-\left(s_{i}^{0}+\alpha T_{i}\right)<R_{i}^{k}, k \geq 1
$$

then the system is not schedulable. Moreover any additional assumption on the system intending to satisfy all the constraints does not have any interest in this case.

\section{Proof}

The proof of this theorem derives directly from the assumption that an operation may only be preempted by those already scheduled. An example is given below (see figure 5).

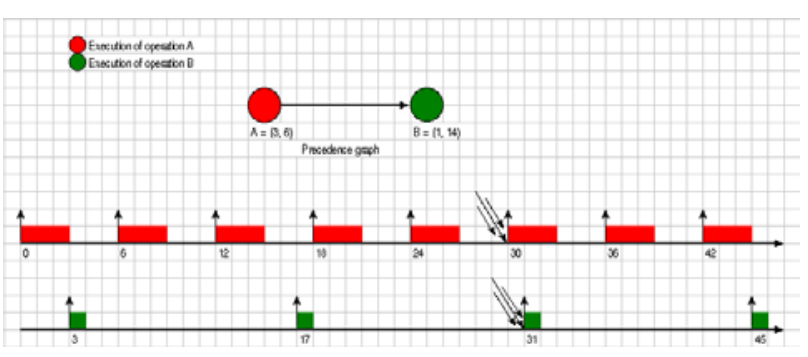

Figure 5. System with an overlapping

Now we can partition $\Omega_{\lambda}$ in the three following subsets: the sub-set $V_{c}$ of systems with overlappings which are not schedulable thanks to theorem 3, the sub-set $V_{d}$ of systems with regular operations, i.e. where the periods of all operations constitute a geometric sequence ${ }^{1}$, and the sub-set $V_{i}$ of systems with irregular operations which both remain potentially schedulable (see figure 6).

$$
\begin{aligned}
V_{c}=\{ & \left\{\left(C_{i}, T_{i}\right)\right\}_{1 \leq i \leq n} \in \Omega_{\lambda} / \forall i \in\{1, \cdots, n-1\}, \\
& \exists j \in\{i+1, \cdots, n\} \text { and } \\
0 \leq & \left.\left(s_{j}^{0}+\beta T_{j}\right)-\left(s_{i}^{0}+\alpha T_{i}\right)<R_{i}^{k}, \quad k \geq 1\right\} \\
V_{d}=\left\{\left\{\left(C_{i}, T_{i}\right)\right\}_{1 \leq i \leq n} \in \Omega_{\lambda} / T_{1}\left|T_{2}\right| \cdots \mid T_{n}\right\} & \\
V_{i}= & \Omega_{\lambda} \backslash\left(V_{c} \cup V_{d}\right)
\end{aligned}
$$

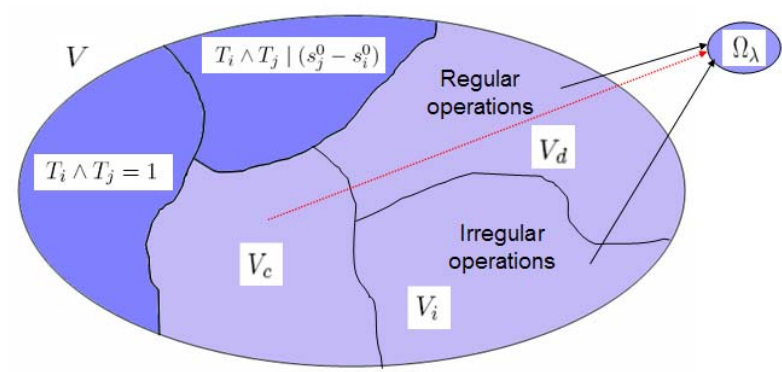

Figure 6. $\Omega_{\lambda}$-partitionning

At this point, we have given non schedulability conditions for systems subject to precedence and strict periodicity constraints. Thus, thanks to the partition of $V$ obtained in figure 6 , once the previous cases discussed above are excluded, the remaining systems of operations corresponding to $V_{d} \cup V_{i}$ are potentially schedulable.

\section{Conclusion and future works}

We are interested in hard real-time systems subject to precedence and strict periodicity constraints where it is mandatory to satisfy these constraints. We are also interested in preemption which offers great advantages when seeking valid schedules. Because the classical approaches are not well suited for the applications we are interested in, we propose a new approach consisting firstly in building

\footnotetext{
${ }^{1}$ A sequence $\left(a_{i}\right)_{1 \leq i \leq n}$ is geometric if and only if it exists $q$ such that $a_{i+1}=q a_{i} \quad \forall i \geq 1$.
} 
a set $V$ of all systems of operations, secondly in restricting $V$ by providing non-schedulability conditions thanks to properties on the strict periods and the WCET of all operations. At the end, these non-schedulability conditions lead to the subset $V_{d} \cup V_{i}$ of systems with both regular and irregular operations as presented in section 3.2 which consists in potentially schedulable systems of operations. The proposed approach restricts drastically the domain of the possible schedules and will lead to a simpler scheduling analysis.

Presently we are seeking schedulability conditions and valid schedules for systems in $V_{d} \cup V_{i}$. Afterwards, we plan to extend our study to the problem with several computing resources.

\section{References}

[1] Joseph Y.-T. Leung and M. L. Merrill. A note on preemptive scheduling of periodic, real-time tasks. Information Processing Letters, 1980.

[2] J.P. Lehoczky, L. Sha, and Y Ding. The rate monotonic sheduling algorithm: exact characterization and average case bahavior. Proceedings of the IEEE RealTime Systems Symposium, 1989.

[3] N.C. Audsley, Burns A., M.F. Richardson, and A.J. Wellings. Hard real-time scheduling : The deadlinemonotonic approach. Proceedings 8th IEEE Workshop on Real-Time Operating Systems and Software, 1991.

[4] Jukka Mäki-Turja, Gerhard Fohler, and Kristian Sandström. Towards efficient analysis of interrupts in real-time systems. 11th EUROMICRO Conference on Real-Time Systems, 1999.

[5] Patchrawat Uthaisombut. The optimal online algorithms for minimizing maximum lateness. Proceedings of the 9th Scandinavian Workshop on Algorithm Theory (SWAT), 2003.

[6] C.L. Liu and J.W. Layland. Scheduling algorithms for multiprogramming in a hard-real-time environment. Journal of the ACM, 1973.

[7] L. Cucu, R. Kocik, and Y. Sorel. Real-time scheduling for systems with precedence, periodicity and latency constraints. In Proceedings of 10th Real-Time Systems Conference, RTS'02, Paris, France, March 2002.

[8] J.H.M. Korst, E.H.L. Aarts, and J.K. Lenstra. Scheduling periodic tasks. INFORMS Journal on Computing 8, 1996.

[9] L. Cucu and Y. Sorel. Schedulability condition for systems with precedence and periodicity constraints without preemption. In Proceedings of 11th Real-Time Systems Conference, RTS'03, Paris, March 2003. 